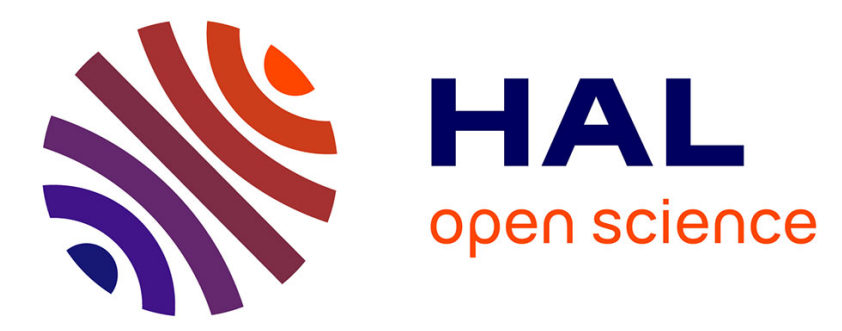

\title{
Structural synergy in a core-shell spin crossover nanoparticle investigated by an electroelastic model
}

Ahmed Slimani, Hamadi Khemakhem, Kamel Boukheddaden

\section{To cite this version:}

Ahmed Slimani, Hamadi Khemakhem, Kamel Boukheddaden. Structural synergy in a core-shell spin crossover nanoparticle investigated by an electroelastic model. Physical Review B, 2017, 95 (17), 10.1103/PhysRevB.95.174104 . hal-03044626

\section{HAL Id: hal-03044626 \\ https://hal.science/hal-03044626}

Submitted on 25 Jun 2021

HAL is a multi-disciplinary open access archive for the deposit and dissemination of scientific research documents, whether they are published or not. The documents may come from teaching and research institutions in France or abroad, or from public or private research centers.
L'archive ouverte pluridisciplinaire HAL, est destinée au dépôt et à la diffusion de documents scientifiques de niveau recherche, publiés ou non, émanant des établissements d'enseignement et de recherche français ou étrangers, des laboratoires publics ou privés. 


\title{
Structural synergy in a core-shell spin crossover nanoparticle investigated by an electroelastic model
}

\author{
Ahmed Slimani, ${ }^{1,}{ }^{*}$ Hamadi Khemakhem, ${ }^{1}$ and Kamel Boukheddaden ${ }^{2, \dagger}$ \\ ${ }^{1}$ Laboratoire des Matériaux Multifonctionnels et Applications, Département de physique, Faculté des Sciences de Sfax, \\ University of Sfax, Route de la Soukra km 3.5 - B.P. n 1171 - 3000, Sfax, Tunisia \\ ${ }^{2}$ Groupe d'Études de la Matière Condensée, CNRS-Université de Versailles, 45 Avenue des Etats Unis, F-78035 Versailles Cedex, France
}

(Received 7 December 2016; revised manuscript received 8 April 2017; published 16 May 2017)

\begin{abstract}
Understanding how surrounding environments act on the functional properties of switchable nano-objects across extended and multiple length scales is of growing interest in many areas of material science. Here, we examine in details, using a microscopic model, the interplay between the structural properties of an inert shell and a spin-active spin-crossover ( $\mathrm{SCO}$ ) core, composed of atoms which can switch thermally between the low-spin (LS) and high-spin (HS) states, a transition which is accompanied with a volume expansion. To come closer to realistic experimental data, we considered a shell having the lattice parameter of the HS state. Intensive Monte Carlo simulations, running on the spin states and atomic positions, are performed on the core-shell spin-crossover nanoparticle using an electroelastic model based on a compressible 2D lattice. A detailed analysis of the effect of the shell's size and rigidity on the magnetostructural properties of the core allows us to address the following issues: (i) the heteroelastic properties of the lattice induce a spatially inhomogeneous pressure (negative in the shell and positive in the core) which strongly distorts the lattice when the core is in the LS state, creating a visible spatial deflection of the shell/core interface; (ii) the thermally-induced first-order SCO transition of the core is significantly affected by the increase of the shell size, which lowers the transition temperature and reduces the thermal hysteresis width; (iii) the shell's rigidity dependence of the thermal hysteresis of the nanoparticle exhibited a resonance behavior when the shell's rigidity equals that of the core, a feature that is analyzed on the basis of acoustic impedance mismatch between the core and the shell. All these outcomes reflect the crucial influence of the surrounding environment on the structural properties of the nanoparticle and provide potentialities in the control of the bistability and cooperativity of the SCO nanoparticles by acting on their shell's rigidity.
\end{abstract}

DOI: 10.1103/PhysRevB.95.174104

\section{INTRODUCTION}

Particularly fascinating are the chemical and physical properties of the switchable molecular solids as the spin transition compounds [1-10]. Up to now, much of the research in this area has been driven to introduce such compounds as nano-objects into a polymer matrix, since it has proven to be very promising in highly technological applications [9,11-37]. The surrounding environment of the spin crossover materials, like the shell in a core/shell structure [29-34] offers the possibility to design and control the spin transition in a way that can go beyond naturally occurring in a free spin crossover nanosystem. Different approaches have been proposed to realize speculative ideas about the impact of the surrounding environment on the yielding of the spin crossover materials [38-48], but a detailed understanding of such relationship between the spin crossover core and the surrounding environment as well as their interplay with structural changes is challenging to unravel. The core-shell nanoparticles display a variety of complex phenomena as a marginal change in the transition temperature, a partial character of the spin transition as well as a significant lattice contraction through the spin transition which induces strain on the shell. Obviously, the response of the spin crossover core at the same time to the external stimuli and to the matrix is a source of interest and controversy. Why is this so? So far, there has been relatively little attention paid to the structural studies on the effects of the

\footnotetext{
*ahmed.slimani@fss.rnu.tn

†kbo@physique.uvsq.fr
}

medium on the features of the nanoparticle's core. Here, we consider a spin crossover nanoparticle from a theoretical point of view using a microscopic electroelastic model [49]. This two-dimensional system was originally designed to mimic a core-shell structure formed by an inner spin-active core with spin crossover properties and an outer spin-inactive shell. The electroelastic model has shown its merits in the analysis of the thermal spin transition of spin crossover nanoparticles [46-48]. The additional insight gained through this study will be a useful step toward the elucidation of the structural relationship between the core and the shell properties. The goal is to reveal which part will experience higher strain and how it is connected to the nanoarchitecture of the nanoparticle. The paper is organized as follows: Section II introduces the electroelastic model and the adopted calculation procedure. The influence of the shell's thickness on the thermal spin transition of the nanoparticle as well as on the structural parameters are analyzed in Sec. III. In Sec. IV, we conclude the paper.

\section{MODEL HAMILTONIAN}

The core-shell nanoparticle was designed in a 2D lattice whose atoms can move only inside the plane. The topology of the system was fixed, where the nodes are placed respecting a square symmetry. Each node has four nearest neighbors (nn) and four next-nearest neighbors (nnn), except those at the surface due to the open boundary condition. To simulate the spin-crossover molecules, each node of the lattice may have two spin states; high-spin (HS) and low-spin (LS) described by the respective eigenvalues +1 and -1 of an associated 
fictitious spin $S$. The core which is here the functional part of the nanoparticle was represented by active spin crossover nodes. In contrast, the shell's molecules surrounding the core are considered as inactive from the magnetic point of view, although the model can be easily adapted to the case of a magnetic shell. To study situations close to the experimental ones, we consider here that the shell has a lattice parameter equal to that of the core in the HS state. Thus, the core's spins and positions could be changed using the usual Monte Carlo procedure, while in the HS shell only the positions are changed in the Monte Carlo simulations. In the core, the equilibrium distances and the interactions between the molecules depend on their spin states. In the present model, the nn and nnn atoms are linked by springs whose stiffness's depend on the instantaneous distance between the nodes. The electroelastic Hamiltonian has the following form,

$$
H=H_{c}+H_{s}+H_{c-s},
$$

where $H_{c}, H_{s}$, and $H_{c-s}$ are respectively, the core, the shell, and the core-shell contributions to the total Hamiltonian (1). Their expressions are given as follows,

$$
\begin{gathered}
H_{c}=\sum_{i \in \text { core }} \frac{\left(\Delta-k_{B} T \ln g\right) S_{i}}{2} \\
+\sum_{i, j \in \text { core }} A_{i j}\left[r_{i j}-R_{0}\left(S_{i}, S_{j}\right)\right]^{2} \\
+\sum_{i, k \in \text { core }} B_{i k}\left[r_{i k}-R_{0}^{\prime}\left(S_{i}, S_{k}\right)\right]^{2}, \\
H_{s}=\sum_{i, j \in \text { shell }} \Gamma\left[r_{i j}-R_{s}\right]^{2}+\sum_{i, k \in \text { shell }} \Lambda\left[r_{i k}-R_{s}^{\prime}\right]^{2},
\end{gathered}
$$

and

$$
\begin{aligned}
H_{c-s}= & \sum_{i \in \text { core }} \frac{\left(\Delta-k_{B} T \ln g\right) S_{i}}{2} \\
& +\sum_{i, j \in \text { core }} A_{i j}\left[r_{i j}-R_{0}\left(S_{i}, S_{j}\right)\right]^{2} \\
& +\sum_{i, j \in \text { shell }} \Gamma\left[r_{i j}-R_{s}\right]^{2}+n n n .
\end{aligned}
$$

The core part Eq. (2) contains active spin-crossover atoms which are subject to the effective energy splitting, composed of the ligand field energy $\Delta$ and the entropic effects $\left(k_{B} T \ln g\right)$ arising from the degeneracy ratio between the HS and LS states. The second and third terms are the elastic contributions, where the quantity $R_{0}\left(S_{i}, S_{j}\right)$ is the equilibrium bond lengths between two nodes $i$ and $j$ which depends on the spin states of the connected bonds. The latter are denoted $R_{0}(+1,+1)=$ $R_{0}^{H H}, \quad R_{0}(+1,-1)=R_{0}(-1,+1)=R_{0}^{\mathrm{HL}}, \quad$ and $R_{0}(-1,-1)$ $=R_{0}^{L L}$, for the HS-HS, HS-LS, and LS-LS site configurations. It is straightforward to demonstrate that

$$
R_{0}\left(S_{i}, S_{j}\right)=\rho_{0}+\rho_{1}\left(S_{i}+S_{j}\right)+\rho_{2} S_{i} S_{j},
$$

where $\rho_{0}=\frac{\left(R_{0}^{H H}+2 R_{0}^{\mathrm{HL}}+R_{0}^{L L}\right)}{4}, \quad \rho_{1}=\frac{\left(R_{0}^{H H}-R_{0}^{L L}\right)}{4}, \quad$ and $\rho_{2}=$ $\frac{\left(R_{0}^{H H}-2 R_{0}^{\mathrm{HL}}+R_{0}^{L L}\right)}{4}$. In the Hamiltonian (2), $A_{i j}\left(S_{i}, S_{j}\right)$ [resp. $\left.B_{i k}\left(S_{i}, S_{k}\right)\right]$ denotes the local bond stiffness of nn (respectively, $\mathrm{nnn})$ bonds and is written under the following form so as to decrease the total elastic constant in the HS spin state; $A_{i j}\left(r_{i j}\right)=A_{0}+A_{1}\left(r_{i j}-R_{0}^{H H}\right)^{2}$ and $B_{i k}\left(r_{i k}\right)=B_{0}+$ $B_{1}\left(r_{i k}-\sqrt{2} R_{0}^{H H}\right)^{2}$. $A_{0}$ (resp. $\left.B_{0}\right)$ and $A_{1}$ (resp. $\left.B_{1}\right)$ are, respectively, the harmonic and the anharmonic contributions to the elastic interaction energy between nn (resp. nnn) neighbors, while $r_{i j}=\left\|\vec{r}_{i}-\vec{r}_{j}\right\|$ (resp. $r_{i k}$ ) is the instantaneous distance between two nn (resp. nnn) sites. We should point out that we are using the anharmonic term to satisfy the reality condition that the LS state has a stronger rigidity (i.e., higher bulk modulus) than the HS state [50,51]. Further, the presence of bond bending interactions in the model is needed to avoid the collapse of the lattice due to the shear stress and so to maintain the symmetry.

As seen in Eq. (3), the shell Hamiltonian contains only elastic energy since the sites of the shell are considered as spin inactive. The sites interact via the elastic constants $\Gamma$ for the $n n$ and $\Lambda$ for the nnn. In addition, the equilibrium distance between the shell atoms is taken equal to $R_{s}$. The latter will be assumed equal to the lattice parameter of the core in the HS state $\left(R_{s}=R_{0}^{H H}\right)$ as already reported in some experimental works [37]. However, in the general case, the equilibrium distance in the shell lattice can be tuned, a situation that we will analyze separately in a forthcoming work.

The last contribution, given in Eq. (4), accounts for the energy of the atoms located at the interface between the core and the shell. So according to their position (in the core or in the shell part), they may have a spin state dependence or not and their elastic interactions with their neighbors goes through $\Gamma$ or $A_{i j}$. In this study, we will mainly use for simplicity, $\Gamma=A_{i j}$ and $\Lambda=B_{i j}$, except in one section where we analyze the effect of the mechanical rigidity of the shell on the thermal switching properties of the core.

Finally, we mention that the present Hamiltonian naturally generates short- and long-range interactions between the spin states [49]. The short-range interactions involve fictitious spin-spin "exchange" interactions through lattice distortions and the long-range interactions arise from the macroscopic volume change. It should nevertheless be pointed out that the "exchange" between the fictitious spin states is not a stricto sensu a magnetic interaction. Magnetic interactions may indeed exist in some rare spin-crossover materials [52,53] and can be modeled using an adapted Blume-Emery-Griffiths model [54].

In our simulations, we have used as far as possible realistic parameter values derived from experimental data. For example, the ligand field energy $\Delta$ is deduced from heat capacity data. Indeed, upon a complete transition between LS and $\mathrm{HS}$, the one site electronic energy change $\Delta$ relates to the molar enthalpy change through $\Delta H=N_{A} \Delta$, where $N_{A}$ is the Avogadro number, since the elastic energies of the HS and LS states are zero at equilibrium. Furthermore, the degeneracy ratio $g$ connects to the molar entropy change as follows: $\Delta S=R \ln g$, where $R$ is the perfect gas constant. In the present simulations, we used the parameter values $\Delta=1500 \mathrm{~K}$ and $g=150$ [55-57], leading to $\Delta H \approx$ $12.5 \mathrm{~kJ} / \mathrm{mol}$ and $\Delta S \approx 42 \mathrm{~J} / \mathrm{K} / \mathrm{mol}$, which are in very good agreement with the experimental calorimetric measurements on archetypal spin crossover compounds whose $\Delta H$ and $\Delta S$

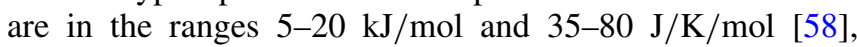
respectively. 
The lattice parameter values in the LS and HS states are, respectively, $R_{0}(-1,-1)=1 \mathrm{~nm}$ and $R_{0}(+1,+1)=1.2 \mathrm{~nm}$ while for the HL state we have simply considered the average value, $R_{0}(+1,-1)=\frac{\left(R_{0}(+1,+1)+R_{0}(-1,-1)\right)}{2}=1.1 \mathrm{~nm}$. According to the square symmetry of the lattice, we imposed the lattice parameter between nnn HS (LS) atoms as given by $\sqrt{2} R_{0}(+1,+1) \approx 1.7 \mathrm{~nm}\left[\sqrt{2} R_{0}(-1,-1) \approx\right.$ $1.4 \mathrm{~nm}$ ]. From the experimental point of view, the bulks modulus of spin crossover materials in the HS and LS states have been derived from Brillouin scattering for a single crystal of $\left[\mathrm{Fe}(p t z)_{6}\right]\left(\mathrm{ClO}_{4}\right)_{2}$ and were estimated in the range 5-20 $\mathrm{GPa}[50,51]$. The values of the respective nn and nnn elastic constants $A_{0}$ were evaluated by considering the elongation of a cubic cell, of lattice parameter $a$, submitted to a uniaxial stress and by neglecting the transverse effects. This simple model leads to the following approximate relation $A_{0}+2 B_{0}=E \times a$, where $E$ is the bulk modulus. Using $A_{0}=50000 \mathrm{~K} / \mathrm{nm}^{2}=50 \mathrm{meV} / \AA^{2}$ leads to reasonable bulk modulus value, $E \approx 12 \mathrm{GPa}$. In addition, the anharmonic nn elastic constant was set equal to $A_{1}=10 A_{0}$, while the harmonic and anharmonic nnn elastic constants are chosen such as $B_{0}=0.28 A_{0}$ and $B_{1}=0.28 A_{1}$, respectively.

Let us now discuss the technical aspects of the simulation. The total Hamiltonian is solved using a two-step iterative strategy running sequentially over the spin and position variables as follows: at each temperature, a single site $i$ in the core part, chosen randomly, of spin $\left(S_{i}= \pm 1\right)$ at position $\vec{r}_{i}$ is checked for a spin change $S_{i} \rightarrow-S_{i}$. The new value of the spin is accepted or rejected by the usual Monte-Carlo metropolis criterion, based on the comparison of the final and initial energies. Then, to ensure the mechanical relaxation, all nodes of lattice (randomly selected in the core and the shell) are asked for a slight displacement by a quantity $\left\|\delta \overrightarrow{r_{i}}\right\| \ll\left\|\overrightarrow{r_{i j}}\right\|$. In the present simulations, we have fixed the amplitude of the random displacements as $\delta r=0.05 \mathrm{~nm}$ which remain quite small compared to the lattice parameter values in the LS and HS spin states which are, respectively, equal to $1 \mathrm{~nm}$ and $1.2 \mathrm{~nm}$. The position update is accepted or rejected using the usual Monte-Carlo metropolis criterion. At this stage, each lattice position has been updated by the Monte-Carlo process $N$ times, where $N$ is the total number of nodes in the lattice (core and shell), defined as $N=L \times L$ with $L$ is the linear size of the nanoparticle. Afterwards, a new spin site in the core is randomly selected and the procedure is repeated $N$ times so as to have a chance to visit each spin site on average in the core. Once we execute $N$ updates on the spin changes, we define such a step as the Monte Carlo step (MCS) unit. In practice, for each temperature, we performed 1000 MCS to reach the thermal equilibrium. Afterwards, another 1000 MCS are utilized to calculate the thermal averages of the physical quantities of interest: average magnetization, average lattice parameter, average elastic energy, etc. As a result, at each temperature during the MC procedure, each spin site is visited $2 \times 1000$ times, while each node position is updated $2 \times N \times 1000$ times. To derive the thermal properties of the core-shell particle, we performed thermal hysteresis studies by changing the temperature between 0 and $400 \mathrm{~K}$ with a thermal increment of $1 \mathrm{~K}$ following the heating and the cooling process.

\section{RESULTS AND DISCUSSION}

For a deeper understanding of the intimate relationships between the shell and the core of spin crossover nanoparticles and how they are mutually intricate, we developed a detailed structural investigation of each apart. For this purpose, we monitored the average properties of a 2D system with squared symmetry during the thermoinduced spin transition by means of HS fraction, $n_{\mathrm{HS}}$, which represents the probability of occupying the HS state at given temperature, defined as

$$
n_{\mathrm{HS}}=\frac{\left(1+\left\langle S_{i}\right\rangle\right)}{2},
$$

as well as through the lattice parameters of the core and the shell, separately, defined as

$$
\langle d\rangle=\left\langle\frac{\sum_{i j} \sqrt{\left(x_{i}-x_{j}\right)^{2}+\left(y_{i}-y_{j}\right)^{2}}}{\frac{n_{b}}{2}}\right\rangle,
$$

where $n_{b}$ is the bonds number between neighboring molecules $i$ and $j$ of coordinates $\left(x_{i}, y_{i}\right)$ and $\left(x_{j}, y_{j}\right)$ and $\langle\ldots\rangle$ stands for the thermal averages.

Let us denote by $N_{c}$ and $N_{s}$ the number of sites in the core and the shell, respectively, and by $N=N_{c}+N_{s}$ the total number of sites in the nanoparticle. The linear size of the core (resp. the thickness of the shell) is denoted $L_{c}$ (resp. $\omega$ ) and the nanoparticle's size is denoted $L=L_{c}+2 \omega$, which straightforwardly depend on the core and shell lattice parameters. In the present simulations, $L$ ranges between 21 and $49 \mathrm{~nm}$. Moreover, the linear size of the core $L_{c}$ was fixed to $19 \mathrm{~nm}$ while the thickness of the shell $\omega$ varies in the range of [1-15] nm.

For the sake of simplicity, we assumed, in the first part of this work, that the shell and the core have the same elastic constants, i.e., $A_{0}=\Gamma$. Further, the equilibrium distances between the neighboring atoms in the shell have been set equal to those of the core in the HS state. As stated previously, this choice reflects experimental works [37], although in the general case, the shell may have its own lattice parameter which can be different from those of the LS and HS states of the core. This interesting case will be studied and reported in a separate work.

\section{A. The thermoinduced spin transition}

To highlight the elastic effects of the shell on the thermodynamic properties of the nanoparticle, we reported in Fig. 1(a) the thermoinduced spin transition of a free core and that of a core-shell system for different shell's thickness. One can easily remark that thick shells affect the thermal hysteresis by (i) shifting the whole hysteresis loops towards low temperature regions and (ii) narrowing their width until (almost) vanishing for a shell's thickness $\approx 15 \mathrm{~nm}$, in good agreement with prior reports [46,48]. The equilibrium temperature $T_{e q}$ and the hysteresis width $\Delta T$ are estimated numerically as

$$
T_{e q}=\frac{T^{\mathrm{HS} \rightarrow \mathrm{LS}}+T^{\mathrm{LS} \rightarrow \mathrm{HS}}}{2}, \Delta T=T^{\mathrm{LS} \rightarrow \mathrm{HS}}-T^{\mathrm{HS} \rightarrow \mathrm{LS}},
$$

where $T^{\mathrm{HS} \rightarrow \mathrm{LS}}$ (resp. $T^{\mathrm{LS} \rightarrow \mathrm{HS}}$ ) is the transition temperature on cooling (resp. heating) from the HS to LS (resp. LS to HS) 

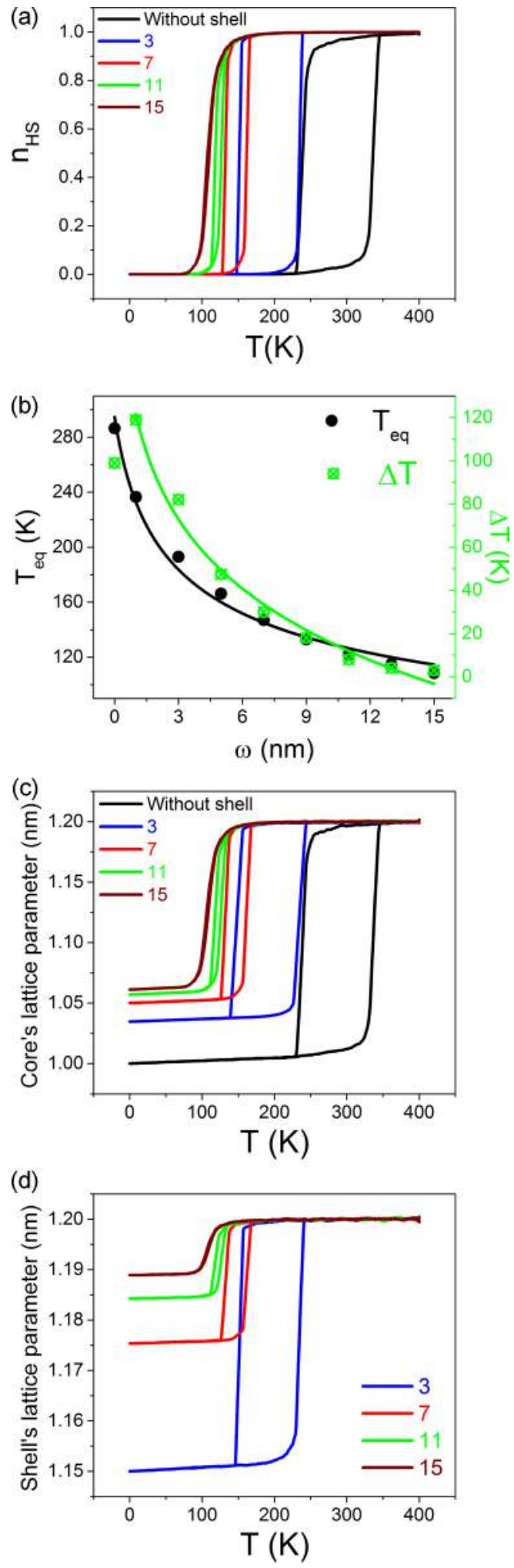

FIG. 1. (a) Thermal evolution of the HS fraction $n_{\text {HS }}$ for different shell thickness. (b) The evolution of the equilibrium temperature $T_{e q}$ (black) and the hysteresis width $\Delta T$ (green) as a function of the shell thickness $\omega$. Thermal variation of the lattice parameters of the core (c) and the shell (d) for the different shell's thickness. The core's linear size is $L_{c}=19 \mathrm{~nm}$. states. Figure 1(b) depicts the shell's thickness dependence of the transition temperature $T_{e q}$ and the thermal hysteresis width $\Delta T$.

A significant change in $T_{e q}$ of about $180 \mathrm{~K}$ is observed between the thinnest $(\omega=1 \mathrm{~nm})$ and the thickest shell $(\omega=$ $15 \mathrm{~nm}$ ). In contrast, the situation is a bit tricky for the hysteresis width, which shows a nonmonotonic behavior with $\omega$. Indeed, compared to the case of the free spin crossover core, one can easily see that $\Delta T$ increases for $\omega=1 \mathrm{~nm}$, corresponding to one monolayer of shell atoms, and falls down for higher values of $\omega$. This behavior is attributed to the mechanical effects exerted by the shell on the core, which will be discussed in more details in the last section.

The concomitant narrowing of hysteresis width $\Delta T$, with the decrease of the transition temperature $T_{e q}$, are consequences of the tensile stress caused by the shell on the core. This can be understood by the fact that the equilibrium lattice parameter of the shell is equal to that of the core in the HS state. In the case of a thin shell, the mechanical effects felt by the spin crossover core atoms are weak but strong enough to promote the HS state, while for a thick shell, the elastic interactions between the spin crossover units are highly hindered by reducing the internal pressures, which weakens the strength of the elastic interactions (i.e., the cooperative character) between the spin transition molecules, resulting in the narrowing of the thermal hysteresis width and even to its disappearance in the limiting cases. Consequently, the LS's lifetime is considerably reduced since the latter state is no longer rigid and therefore the internal elastic forces vanish. It is worth noticing that similar behavior was observed experimentally on $\mathrm{Fe}$ (pyrazine $) \operatorname{Pt}(\mathrm{CN})_{4}$ nanoparticles coated with a silica film with a controlled thickness, for which the authors reported a large (resp. small) hysteresis for thin (resp. thick) shells [30].

\section{B. The structural properties}

As we will describe below, the problem is more about the interplay between the structural parameters of the core and the shell. Thus, to further understand the underlying mechanisms between the core and the shell, we followed separately the thermal variation of their lattice parameters in Figs. 1(c) and 1(d), respectively. One can easily notice a distinct difference in the shape of hysteresis loops of $n_{\mathrm{HS}}$ [Fig. 1(a)] and lattice parameters. Indeed, Figs. 1(c) and 1(d) reveal the presence of residual high temperature lattice parameters at low temperature that has not been observed in the hysteresis loops of the high spin fraction, $n_{\mathrm{HS}}$. Furthermore, these residual high temperature lattice parameters increase with the thickness. In Fig. 2(a), we have monitored separately the low temperature lattice parameters of the core and the shell as functions of the shell's thickness. The variation between the thinnest and the thickest shells at $0 \mathrm{~K}$ in the lattice parameters are estimated as $6 \%$ and $11 \%$ for the core and the shell, respectively. The more the shell is thin the more is impacted by the volume shrinkage of the core and its lattice parameter approaches that of the core's LS state. However, the situation is reversed when 

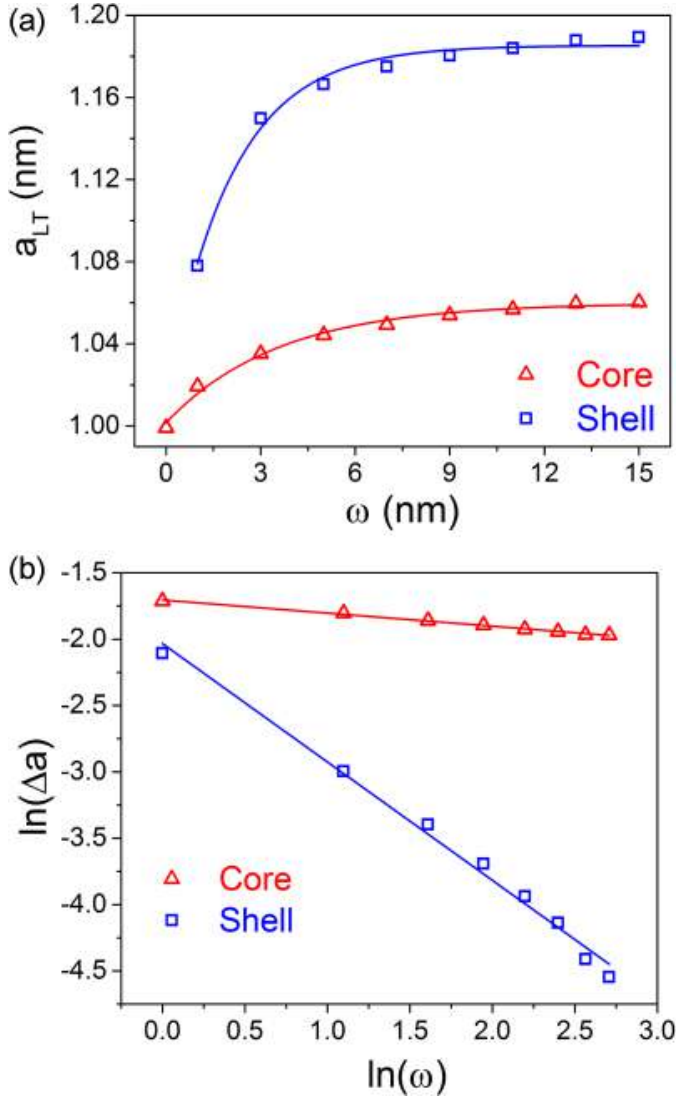

FIG. 2. (a) The shell thickness dependence of the mechanicallyrelaxed low temperature lattice parameters, $a_{L T}$, of the core (red) and the shell (blue). (b) The linear dependence of the logarithm of lattice parameter misfit, $\Delta a=a_{\mathrm{HS}}-a_{L T}$, with the logarithm of the shell's thickness $\omega$.

considering thick shells, the core part is thereby influenced and its LS lattice parameter tends to the shell's one.

The dependency between the misfit of lattice parameters between the HS and LS states $\Delta a=\left(a_{\mathrm{HS}}-a_{L T}\right)$ and the shell's thickness $\omega$ was best described by a $\ln -\ln$ plot reported in Fig. 2(b). Indeed, a thicker shell expands the LS lattice by hindering the elastic interactions between the spin-crossover units and reducing all the internal pressures and the elastic stresses. Thus, the LS state is no more rigid and resembles the HS state, and therefore the internal elastic forces responsible for the cooperative nature of the transition vanish. This is particularly true in the case of a massive shell since the volume ratio (core/shell) become negligible. These facts stabilize the HS state as proved by the shift of $T_{e q}$ towards low temperature as well as the narrowing of the hysteresis loop.

\section{The mechanical properties}

This section is devoted to the study of the mechanical properties of the core-shell nanoparticle to shade light on the interplay between the elastic properties of the shell and the magnetic behavior of the core through "magnetoelastic" interaction. As in the previous sections, the simulations are conducted on a 2D lattice, and one may legitimately ask on the relevance of such approach to describe the real core-shell switchable nanoparticles which are three-dimensional lattices.
In fact, the 2D approach constitutes, indeed, an oversimplified description which has the merit to be tractable numerically, although it masks several 3D effects. As a comparison, the main differences between 3D and 2D systems will arise from: (i) the size effects because the ratio, shell/core is $\left[\left(\frac{L}{L_{c}}\right)^{2}-1\right]$ for a $2 \mathrm{D}$ system where it is $\left[\left(\frac{L}{L_{c}}\right)^{3}-1\right]$ for a $3 \mathrm{D}$, (ii) as well as from the ratio surface/volume which is $\frac{4}{L}-\frac{1}{L^{2}}$ at $2 \mathrm{D}$ and $\frac{6}{L}-\frac{12}{L^{2}}$ at $3 \mathrm{D}$. Because of the open boundaries conditions used in the present problem, the size effects and the dimensionality of the system is expected to play an important role. From the simulations viewpoint, the study of 3D elastic systems has been performed by one of the authors for interacting spin-crossover sheets [59] in order to study the buckling and crumpling effects created by the volume change upon spin crossover transitions. The simulations could have been achieved only for small systems, because they are highly time consuming. So to avoid such complications, we accept the sacrifice to mimic the SCO nanoparticle using a 2D elastic model, which also captures the essential features of the problem.

\section{The local pressure}

The snapshots and the distribution of the local pressure at low temperature were considered in relation to shell's thickness dependency of the thermal behavior of the nanoparticle. The local pressure $P(i)$ of site $i$ is determined as

$$
\begin{aligned}
P(i)= & -\sum_{j} A_{i j}\left(r_{i j}\right)\left(r_{i j}-R_{0}\left(S_{i}, S_{j}\right)\right) \\
& -\sum_{k} B_{i k}\left(r_{i k}\right)\left(r_{i k}-R_{0}\left(S_{i}, S_{k}\right)\right),
\end{aligned}
$$

where $j$ (resp. $k$ ) runs over the nearest (resp. next-nearest) neighbors of site $i$. The snapshot of the lattice corresponding to the low temperature stable state is reported in Fig. 3(a) and is marked by a stressed structure due to the shrinkage of the spin crossover core in the LS state which draws thereby the enveloping shell.

In view of our findings, the analysis of the spatial distribution of the local pressure becomes necessary to investigate the means by which these stresses are structured. The distribution of the local pressure is illustrated in Fig. 3(b) and arises positive pressure area in the core part and negative pressure area in the shell. Indeed, the pressure is inhomogeneously distributed in the lattice, that is, over the shell we distinguish null and negative pressure area (tensile stresses) at the corner and the border(due to the bending effect), while over the core the positive pressure (compressive stresses) peaked at the corner (due to constriction effect) and relatively reduced around the border.

The elastic pressure effects are quantified by evaluating the pressure density $D$ over the whole lattice, given by $D=\frac{\sum_{i} P(i)}{N}$, and which corresponds to the average value of the local pressures at each lattice node. Figure 3(c) displays the pressure density $(D)$ over the core (in red) and the shell (in blue), separately, as functions of the shell's thickness $\omega$. One can notice that the pressure density in the core part increases with the shell's thickness unlike in the shell where the absolute $\mathrm{D}$ decreases. The pressure density in the core and the shell are found to follow a power law as a function of the thickness $\omega$ 
(a)

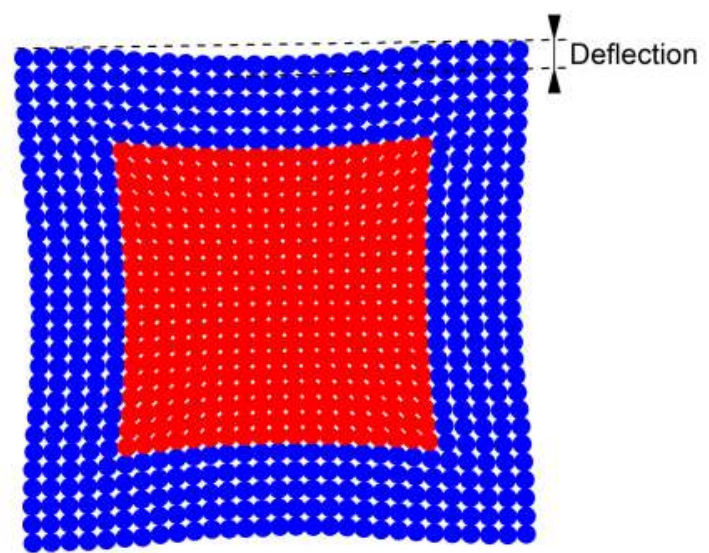

(c)

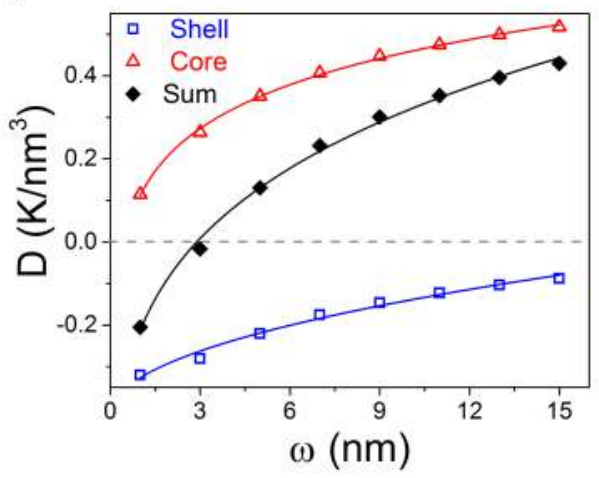

(b)

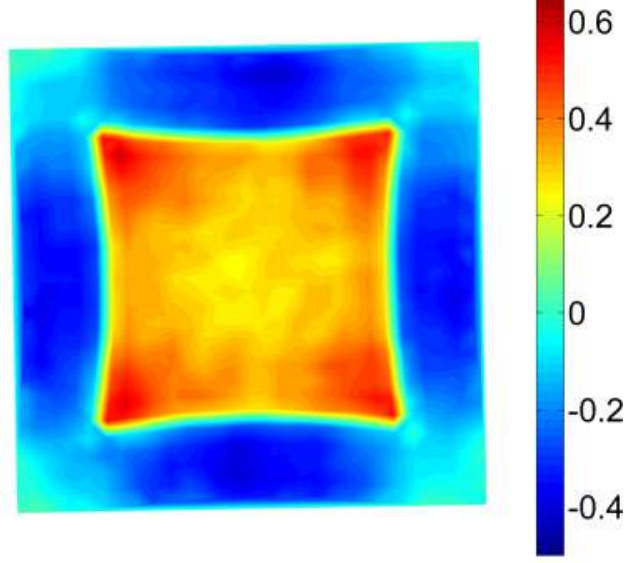

(d)

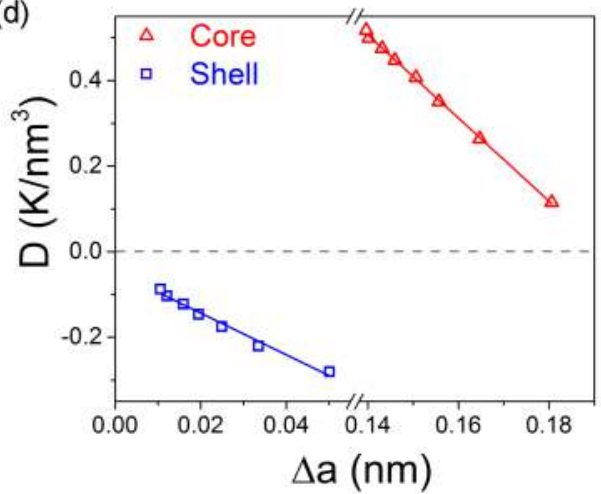

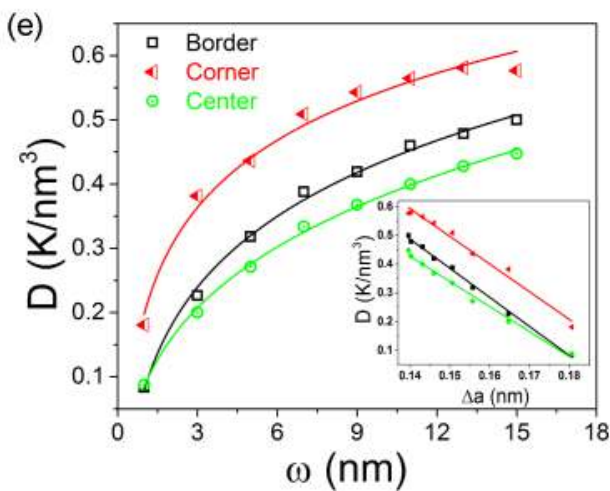

FIG. 3. (a) Snapshot of the LS configuration for a shell thickness of $5 \mathrm{~nm}$. (b) The corresponding distribution of pressure over the nanoparticle presented in (a). (c) The pressure density over the lattice (black), the core (red), and the shell (blue) as a function of the thickness of the shell. (d) Linear correlation between the misfit of lattice parameters $\Delta a$ and the pressure density. (e) The local pressure density over the core at the corner, border, and center. Inset: The correlation between the misfit of lattice parameters and the local pressure density. The presented data have been obtained at $0 \mathrm{~K}$.

as $D=a+b \omega^{c}$. This means that the distribution of pressure over the core and the shell is similar regardless of the core and the shell. It is important to note that the power exponent $c$ is quite different between the core and the shell, $c$ in the core worth 0.48 while in the shell is 0.046 . This significant $\operatorname{contrast}(\approx 10 \%)$ emphasizes the strong dependence of the core's structural parameter on the shell's thickness much as has been observed with the shell itself.

One may expect that the distribution of the local pressure will be balanced over the lattice and, hence the pressure density will be compensated between the core and the shell. But the sum of $D$ over the lattice, presented in black in Fig. 3(c), shows that it is not the case. As we will describe below, the problem could be considered as an interplay of internal forces (from the core) and the external forces (from the shell) that shapes the structural parameters of the lattice. At low temperature (the LS state of the core), the internal and the external forces are out of equilibrium. The internal elastic forces originating from the core are dominant over the shell ones due to the quick release of the excess of elastic energy from the free border of the shell unlike the constrained spin crossover core. Further, the sum of pressure, $D$, reveals that for a shell's thickness 
$\approx 3 \mathrm{~nm}$ the pressure is well balanced over the lattice, which means that $D_{\text {core }}+D_{\text {shell }}=0$, where $D_{\text {core }}$ and $D_{\text {shell }}$ are, respectively, the core and the shell pressure densities. By looking back to Eq. (9) it is easy to note that the local pressure is proportional to the square root of the elastic energy, that is, justify the correlation between the pressure density (D) in the core and the shell with the misfit of lattice parameters $\Delta a=$ $a_{\mathrm{HS}}-a_{L T}$ between the HS and LS states reported in Fig. 3(d).

Detailed investigation of the pressure density on different areas of the core was as well conducted and results are illustrated in Fig. 3(e). The pressure density was averaged in the center, border, and corner of the core using a small region of $5 \times 5 \mathrm{~nm}$. The pressure density has the same behavior as a function of the shell's thickness for the three areas. D is mainly localized on the corner of the core and relatively decreases on the core's border and the minimum was found in the center. In the inset of Fig. 3(e), we verified the linear correlation between the local pressure density and the misfit of lattice parameters. This dependency is related to the elastic interactions within the lattice that the corresponding elastic energy peaks at the HS/LS interface $[60,61]$.

\section{The mechanical interplay between the core and the shell}

The mechanical deformation of the lattice observed at $0 \mathrm{~K}$ was investigated through the analysis of the deflection of the lattice. The 2D lattice consists in a stacking of parallel columns (or rows). In the core's HS state, where there is no elastic misfit between the core and the shell, these columns are parallel and adopt a straight shape. In contrast, when the core becomes LS due to the phase transition, a spontaneous stress is generated between the core and the shell (due to core-shell lattice misfit) resulting in a twisting of the atomic lines (the columns), as it appears in Fig. 3(c). The degree of twisting can be measured on the mechanically relaxed lattice by evaluating its deflection. For a "vertical" atomic line of index $i$, along the $y$ direction, the deflection is estimated from the determination of the maximum deformation of the column along the $y$ axis, which corresponds to the difference between the maximum and minimum $x$ coordinates of the column. Within this definition, the deflection is a strictly positive quantity. In the present situation, and due to the symmetry of the lattice, these two points correspond to sites located in the extremity and the center (or vice-versa) of the considered atomic line. However one should mention that the previous definition can be also applied by considering rows instead of columns.

So for the present problem, the deflection $D_{f}$ of a column $i$ is approximately given by:

$$
D_{f}^{(i)}(x)=\left|\frac{x(i, 1)+x(i, L)-2 x\left(i, \frac{L}{2}\right)}{2}\right| .
$$

The deflection term, so defined for a given column $i$, accounts for the interplay between the elastic properties of the shell and the core. Figure 4(a) summarizes the spatial profile of deflection along the $x$ axis over the lattice for a thickness $\omega=15 \mathrm{~nm}$. We observe that the deflection peaks at the vicinity of the core-shell interface, as a result of the volume contraction of the core in the LS state, which pulls the shell towards the lattice's center. Furthermore, the spatial profile of the deflection shows that the latter falls down rapidly
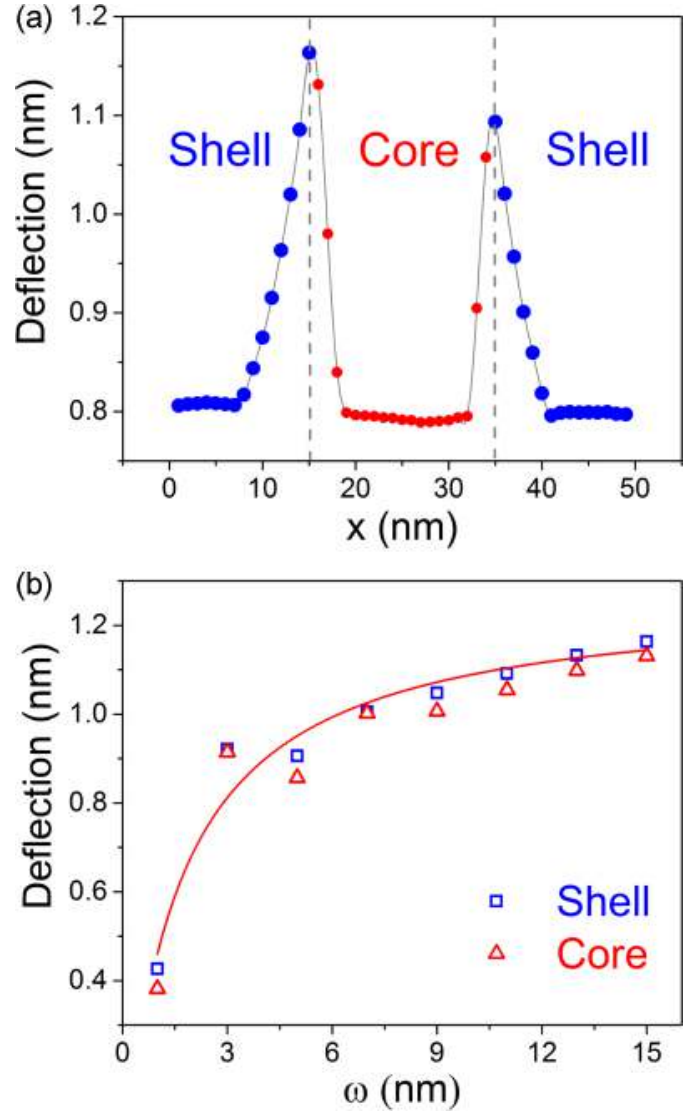

FIG. 4. (a) Deflection profile over the lattice for a shell's thickness of $15 \mathrm{~nm}$. (b) Mechanical deflection of the core/shell interface for the different shell's thickness. The presented data have been obtained at $0 \mathrm{~K}$.

by almost $\approx 2.5 \mathrm{~nm}$ (resp. $\approx 7.5 \mathrm{~nm}$ ) for the core (resp. the shell) represented with red (resp. blue) points, away from the maximum value. This asymmetric shape of the deflection profile around the interface is clearly the result of the stronger rigidity of the LS core compared to that of the shell. Moreover, one can also easily remark in Fig. 4(a) that the deflection curve is not symmetric for the left and the right core-shell interfaces, contrary to what we would expect for the system's symmetry. This behavior is attributed to an incomplete mechanical relaxation of the left part of the lattice, due to the stochastic nature of the technique, as it appears in the pressure distribution of Fig. 3(b), where the left top and left bottom corners of the lattice display a slightly stronger internal pressure than the right corners.

In Fig. 4(b), we monitored the deflection of the core/shell interface as a function of the shell's thickness from both sides of the interface. The more the shell is thick the more the deflection increases until it roughly saturates at $\approx 1.1 \mathrm{~nm}$. The latter value of deflection could be considered as the limit of the mechanical stress of the lattice.

We should mention that the mechanical interplay between the core and the shell is particle-size dependent. Thus, we considered a square core-shell nanoparticle whose core's linear size is ranging between 5 and $17 \mathrm{~nm}$ embedded in a shell with different thickness [0-5] $\mathrm{nm}$. The corresponding thermoinduced spin transitions are summarized in Figs. 5(a) and 5(b). In Fig. 5(a), we reported the hysteresis loops of 

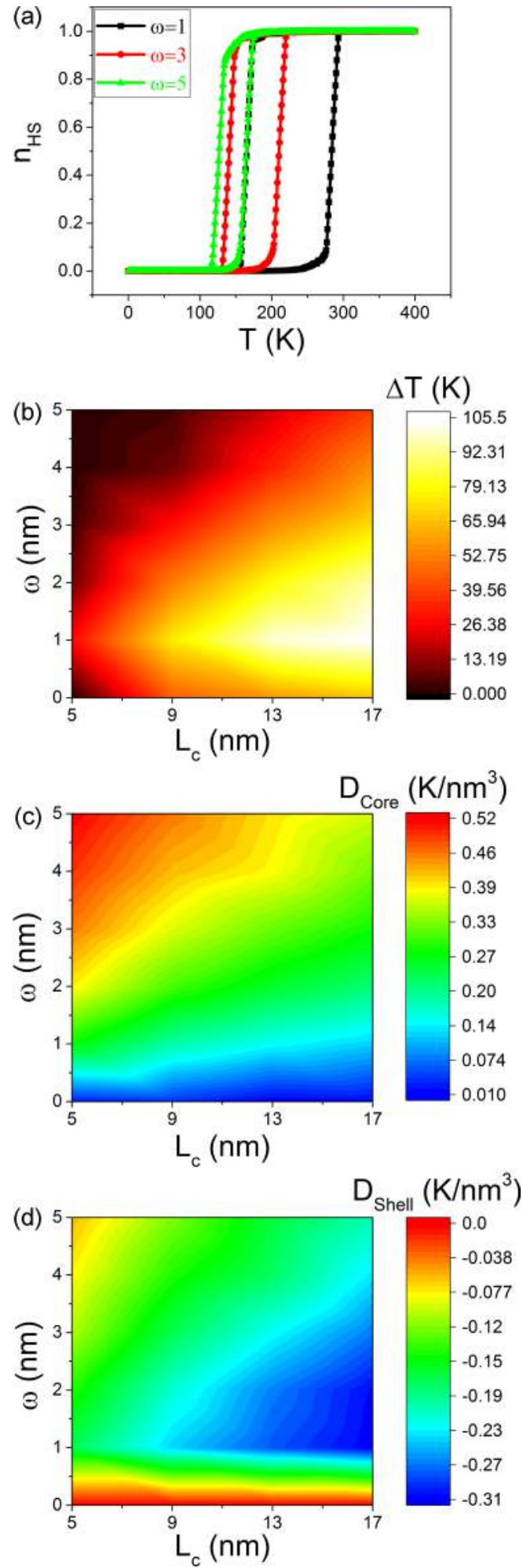

FIG. 5. (a) Effect of the shell's thickness $\omega$ on the thermoinduced spin transition of a square-shaped core of length $13 \mathrm{~nm}$. Diagrams of hysteresis width (b), the pressure density at $0 \mathrm{~K}$ over the core (c), and the shell (d) as functions of the core's linear size and shell's thickness.

a core of linear size $L_{c}=13 \mathrm{~nm}$ embedded in different shell's thickness. The thick shell promotes the HS state, where the hysteresis loops shift towards low temperature with a narrowing in their width.
The widths of the hysteresis loops for the different coreshell nanoparticles are illustrated in Fig. 5(b) and point out an intriguing relation between the core's size and the shell's thickness. The most remarkable feature is that the width of the hysteresis loop is not monotonously dependent on the shell's thickness. Indeed, for all the core sizes, the hysteresis width decreases when the thickness $\omega$ increases, except for $\omega=1 \mathrm{~nm}$ where $\Delta T$ increases and reaches its maximum at $105.5 \mathrm{~K}$. This sharp increase in the hysteresis's width is in good agreement with the observation reported earlier in Fig. 1(b) for a spin crossover core embedded in a thin shell. Such a fact is assigned to the interplay of the mechanical effects between the shell and the core, which become obvious where the ratio $\frac{\text { Shell }}{\text { core }}$ becomes dominant. A result that is confirmed by the negative values of the pressure density in the shell emphasizing the inertia of the shell imposed to the SCO core.

To review the origin of this behavior, we plotted in Figs. 5(c) and 5(d) the diagram of the pressure density over the core and the shell as functions of the core's linear size $L_{c}$ and shell's thickness. The pressure density of the core increases with the shell's thickness and peaks for small cores embedded in thick shells. In the latter situation, the surrounding environment generates a considerable inertia that hinders the volume change accompanying the spin transition. Remarkably, the diagrams of $\Delta T$ and the pressure density of the shell [see Fig. 5(d)] are inversely correlated. By comparing Figs. 5(b) and 5(d), one can easily notice that the largest hysteresis loop corresponds to the most negative pressure density over the shell for $\omega=1 \mathrm{~nm}$ and a core's linear size $L_{c}=17 \mathrm{~nm}$. The diagrams presented in Fig. 5 highlight the key role of the shell on the functional features of the core.

\section{The resonant system}

It is also believed that the stiffness of the surrounding materials may play a crucial role on the functional properties of the core. So that, we investigated in Figs. 6(a)-6(c) the thermoinduced behavior of a core $\left(L_{c}=19 \mathrm{~nm}\right)$ embedded in a shell ( $1 \mathrm{~nm}$ of width) whose stiffness, denoted here $\Gamma$, was varied between $[1-60] \times 10^{4} \mathrm{~K} / \mathrm{nm}^{2}$, while the core's stiffness, $A_{0}$, was fixed at $5 \times 10^{4} \mathrm{~K} / \mathrm{nm}^{2}$. We monitored for the different shell's stiffness the equilibrium temperature $T_{e q}$ and the hysteresis width $\Delta T$ in Fig. 6(a) and the pressure densities over the core, shell, and lattice in Fig. 6(b). For quick convergence in this part, we reduced the MCS steps to 100 for each temperature. According to the value of shell elastic constants $\Gamma$, two regimes could be identified; one for the soft shells $\left(\Gamma \leqslant A_{0}\right)$ and one for rigid shells $\left(\Gamma>A_{0}\right)$. Interestingly, the pressure density in the core increases for the soft shells, while we naively expected that when the core is surrounded by soft materials, it evacuates easily the pressure excess. At this point, it is appropriate to come back to Fig. 3(b) to examine how the pressure is distributed over the core. One can easily see that the pressure is mainly localized in the corner of the core. Indeed, soft shells are easily compressible, and thus the deflection of the nanoparticle's surface is significant. Consequently, the corners of the core are more pinched, leading to the rise of pressure over the core part. Such a constrained situation promotes the LS state which justifies the narrowing of the hysteresis loop for the weak shell's stiffness. 

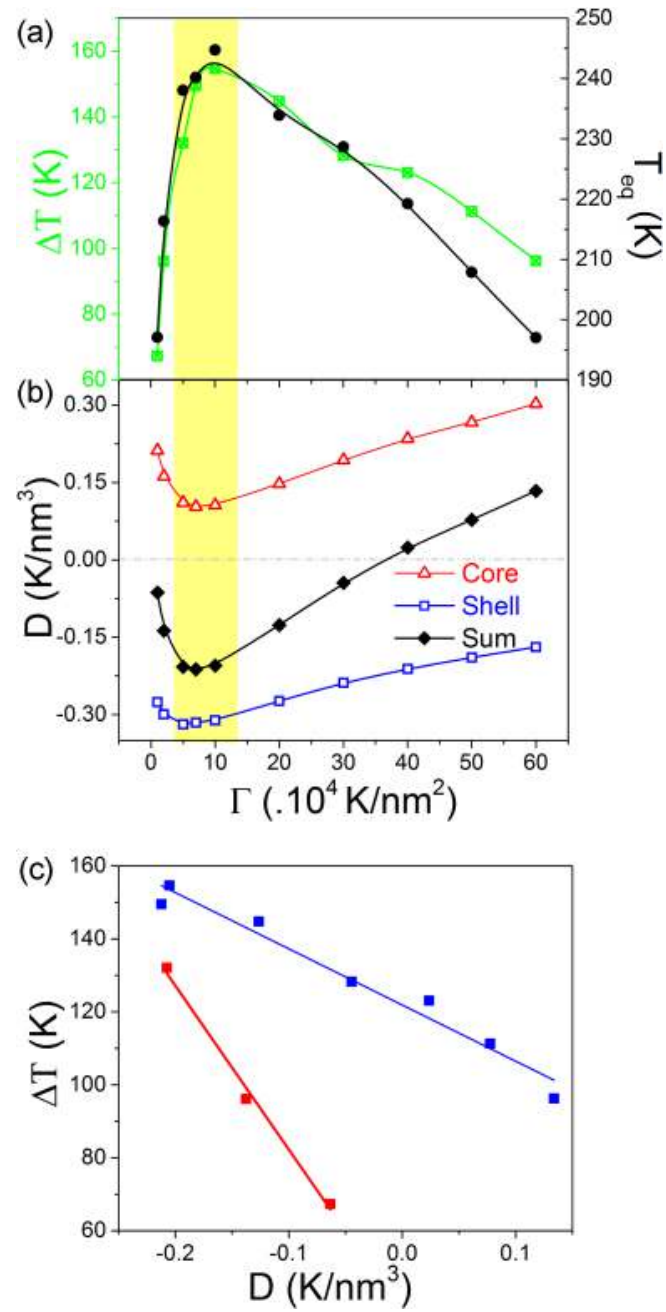

FIG. 6. Effect of the shell's stiffness $\Gamma$ on the (a) thermoinduced spin transition of a core-shell nanoparticle; $\Delta T$ (green) and $T_{e q}$ (black), and (b) pressure density over the lattice (black), the core (red), and the shell (blue) at $0 \mathrm{~K}$. (c) The correlation between the pressure density over the lattice and the hysteresis width for soft shells ( $\left.\Gamma \leqslant A_{0}\right)$ in red and rigid shells $\left(\Gamma>A_{0}\right)$ in blue. (Computed for a shell's thickness $\omega=1 \mathrm{~nm}$, a core's linear size $L_{c}=19 \mathrm{~nm}$, and $A_{0}=5 \times 10^{4} \mathrm{~K} / \mathrm{nm}^{2}$.)

For rigid shells, the situation is more simple since it is observed that the absolute pressure density of the shell decreases while the pressure density increases in the SCO core, where both $T_{e q}$ and $\Delta T$ decrease.

A key point to note from this study is that by adjusting the stiffness of the shell we are able to control the thermal properties of the SCO core. More interestingly is the nonmonotonous behavior of $T_{e q}$ and $\Delta T$ with the mechanical properties of the shell. It is noticed that $T_{e q}$ and $\Delta T$ peak for a shell's stiffness close to the core's one, where the pressure density goes through its absolute maximum in the shell in good agreement with the results reported in Fig. 5.

This behavior can also be analyzed on the basis of acoustic impedance mismatch between the core and the shell. At the interface between two materials of different acoustic impedances, sound waves will be partially reflected and partially transmitted, leading to significant mechanical destruction in materials with strong impedance mismatch [62]. In contrast in materials with low impedance mismatch, the reflection of the sound waves will be negligible, and the elastic interactions (acoustic wave) will propagate much more efficiently in the two media. In the studied nanoparticle here, the volume contraction of the core, due to the HS $\rightarrow$ LS transition, generates an elastically constrained structure with an important pressure density stored in the core. The core's pressure is evacuated easily through a shell with similar elastic features than through a relatively very soft or very stiff shells, leading to enhance the thermal hysteresis width of the SCO core. The direct relationship between the hysteresis width and pressure density of the lattice is validated by a perfect correlation shown in Fig. 6(c).

\section{CONCLUSION}

We have investigated the thermoinduced spin transition of a spin crossover nanoparticle in a core-shell structure using the electroelastic model. The thermal properties of the spin crossover core were correlated with structural and mechanical features of the core-shell nanoparticle. The spin crossover core has clearly demonstrated an environment-dependent behavior as to be responsive to the surrounding environment pressure. Our findings agree well with the experimental observations and the anterior reports. Based on the microscopic nature of the electroelastic model, we have carried out a detailed analysis of structural parameters of the core and shell parts, separately. We demonstrated that a thick shell hinders significantly the elastic interactions between the spin crossover units leading to a rise of the low temperature lattice parameter of the core and the shell as well. By studying the local pressure over the lattice, we found that negative and positive pressure areas are associated with the shell and the core, respectively, because of the intimate coupling of the core and shell that is a result of the heteroelastic features. The pressure density as well as the thermal properties of the core-shell nanoparticle were investigated by varying the core/shell surface ratio. It is evidenced that the width of the hysteresis loop is highly dependent on the internal pressure and the mechanical properties of the shell. Thus, the hysteresis width reaches its maximum at the highest values of the pressure density (in absolute value) in the shell. All observed features amount to the effect of shell's stiffness. We demonstrated that the more homogeneous nanoparticle's stiffness (core and shell), the less constrained core and the larger is the thermal hysteresis width. In particular, by adjusting the shell's stiffness, we were able to control the cooperativity, the thermal properties, and even the width of the thermal hysteresis of the nanoparticle. Such interesting features point out the capacity of control of the spin crossover core through its surrounding environment and beyond its intrinsic properties. These findings may orientate chemists to incorporate the mechanical effect in the elaboration process of the nanoparticle in order to design technologically desirable physical properties. Similar effects could be planned for heterogeneous systems and metamaterials containing photo- or electrosensitive layers to trigger new SCO properties. Finally, one should remark that the lattice symmetry (CFC, CC, triangular, or hexagonal) may play an important role on the thermal properties of the lattice, since the density of nodes are influenced. Thus for a triangular lattice, for example, each site connects to six neighbors while in the hexagonal one, each site has three neighbors. As a consequence, the elastic energy stored in each node will be different, which is then 
expected to affect the core shell properties. This may constitute an interesting extension of the current work.

\section{ACKNOWLEDGMENTS}

This work was supported financially by CNRS ("Centre National de la Recherche Scientifique"), the Université de
Versailles Saint-Quentin, the Université Paris-Saclay, the Université de Sfax, the French Ministère des Affaires Etrangères (Institut Français de Tunisie), as well as the Agence Nationale de la Recherche (Project No. BISTA-MAT: ANR-12-BS070030-01), which are deeply acknowledged. The authors are also indebted to referees of PRB who have contributed by their remarks and criticisms to enhance the value of the paper.
[1] Topics in Current Chemistry, Spin Crossover in Transition Metal Compounds I-III, edited by P. Gütlich and H. A. Goodwin (Springer, Heidelberg, Berlin, 2004), pp. 233-235.

[2] P. Gütlich, Struct. Bonding (Berlin) 44, 83 (1981).

[3] P. Gütlich, A. Hauser, and H. Spiering, Angew. Chem., Int. Ed. Engl. 33, 2024 (1994).

[4] E. König, Struct. Bonding (Berlin) 76, 51 (1991).

[5] O. Kahn and C. J. Martinez, Science 279, 44 (1998).

[6] A. Bousseksou, K. Boukheddaden, M. Goiran, C. Consejo, M.-L. Boillot, and J.-P. Tuchagues, Phys. Rev. B 65, 172412 (2002).

[7] W. Kosaka, K. Nomura, K. Hashimoto, and S. Ohkoshi, J. Am. Chem. Soc. 127, 8590 (2005).

[8] D. Papanikolaou, W. Kosaka, S. Margadonna, H. Kagi, S. Ohkoshi, and K. Prassides, J. Phys. Chem. C 111, 8086 (2007).

[9] F. Prins, M. Monrabal-Capilla, E. A. Osorio, E. Coronado, and H. S. J. van der Zant, Adv. Mater. 23, 1545 (2011).

[10] A. Bousseksou, F. Varret, M. Goiran, K. Boukheddaden, and J.-P. Tuchaguess, Top. Curr. Chem. 235, 65 (2004).

[11] C. Lefter, S. Rat, J. S. Costa, M. D. Manrique-Juarez, C. M. Quintero, L. Salmon, I. Séguy, T. Leichle, L. Nicu, P. Demont, A. Rotaru, G. Molnar, and A. Bousseksou, Adv. Mater. 28, 7508 (2016).

[12] C. Lefter, V. Davesne, L. Salmon, G. Molnar, P. Demont, A. Rotaru, and A. Bousseksou, Magnetochemistry 2, 18 (2016).

[13] J. Dugay, M. Giménez-Marqués, T. Kozlova, H. W. Zandbergen, E. Coronado, and H. S. J. van der Zant, Adv. Mater. 27, 1288 (2015).

[14] A. Holovchenko, J. Dugay, M. Giménez-Marqués, R. TorresCavanillas, E. Coronado, and H. S. J. van der Zant, Adv. Mater. 28, 7228 (2016).

[15] E. Coronado, J. R. Galán-Mascarós, M. Monrabal-Capilla, J. García-Martínez, and P. Pardo-Ibáñez, Adv. Mater. 19, 1359 (2007).

[16] J. R. Galán-Mascarós, E. Coronado, A. Forment-Aliaga, M. Monrabal-Capilla, E. Pinilla-Cienfuegos, and M. Ceolin, Inorg. Chem. 49, 5706 (2010).

[17] A. Rotaru, F. Varret, A. Gindulescu, J. Linares, A. Stancu, J. F. Létard, T. Forestier, and C. Etrillard, Eur. Phys. J. B 84, 439 (2011).

[18] T. Forestier, S. Mornet, N. Daro, T. Nishihara, S. Mouri, K. Tanaka, O. Fouche, E. Freysz, and J.-F. Létard, Chem. Commun., 4327 (2008).

[19] T. Forestier, A. Kaiba, S. Pechev, D. Denux, P. Guionneau, C. Etrillard, N. Daro, E. Freysz, and J.-F. Létard, Chem. Eur. J. 15, 6122 (2009).

[20] L. Salmon, G. Molnar, D. Zitouni, C. Quintero, C. Bergaud, J.-C. Micheau, and A. Bousseksou, J. Mater. Chem. 20, 5499 (2010).
[21] I. Boldog, A. B. Gaspar, V. Martínez, P. Pardo-Ibañez, V. Ksenofontov, A. Bhattacharjee, P. Gütlich, and J. A. Real, Angew. Chem., Int. Ed. 47, 6433 (2008).

[22] C. Bartual-Murgui, N. A. Ortega-Villar, H. J. Shepherd, M. C. Munoz, L. Salmon, G. Molnar, A. Bousseksou, and J. A. Real, J. Mater. Chem. 21, 7217 (2011).

[23] J. Larionova, L. Salmon, Y. Guari, A. Tokarev, K. Molvinger, G. Molnar, and A. Bousseksou, Angew. Chem., Int. Ed. 47, 8236 (2008).

[24] A. Tissot, C. Enachescu, and M.-L. Boillot, J. Mater. Chem. 22, 20451 (2012).

[25] F. Volatron, L. Catala, E. Rivière, A. Gloter, O. Stéphan, and T. Mallah, Inorg. Chem. 47, 6584 (2008).

[26] M. Giménez-Marqués, M. Luisa Garcia-Sanz de Larreaa, and E. Coronado, J. Mater. Chem. C 3, 7946 (2015).

[27] H. Peng, S. Tricard, G. Félix, G. Molnar, W. Nicolazzi, L. Salmon, and A. Bousseksou, Angew. Chem. 126, 11074 (2014).

[28] E. J. Devid, P. N. Martinho, M. V. Kamalakar, I. Salitro, U. Prendergast, J. F. Dayen, V. Meded, T. Lemma, R. GonzalezPrieto, F. Evers, T. E. Keyes, M. Ruben, B. Doudin, and S. J. van der Molen, ACS NANO 9, 4496 (2015).

[29] D. Mader, S. Pillet, C. Carteret, M. J. Stebe, and J. L. Blin, Journal of Dispersion Science and Technology 32, 1771 (2011).

[30] Y. Raza, F. Volatron, S. Moldovan, O. Ersen, V. Huc, C. Martini, F. Brisset, A. Gloter, O. Stéphan, A. Bousseksou, L. Catala, and T. Mallah, Chem. Commun. 47, 11501 (2011).

[31] S. Titos-Padilla, J. M. Herrera, X.-W. Chen, J. J. Delgado, and E. Colacio, Angew. Chem., Int. Ed. 50, 3290 (2011).

[32] J. M. Herrera, S. Titos-Padilla, S. J. A. Pope, I. Berlanga, F. Zamora, J. J. Delgado, K. V. Kamenev, X. Wang, A. Prescimone, E. K. Brechinf, and E. Colacio, J. Mater. Chem. C 3, 7819 (2015).

[33] I. Suleimanov, J. S. Costa, G. Molnar, L. Salmon, and A. Bousseksou, Chem. Commun. 50, 13015 (2014).

[34] D. Qiu, L. Gu, X. L. Sun, D. H. Ren, Z. G. Gu, and Z. Li, RSC Adv. 4, 61313 (2014).

[35] D. Tanaka, N. Aketa, H. Tanaka, T. Tamaki, T. Inose, T. Akai, H. Toyama, O. Sakata, H. Tajiri, and T. Ogawa, Chem. Commun. 50, 10074 (2014).

[36] C. Bartual-Murgui, E. Natividad, and O. Roubeau, J. Mater Chem. C 3, 7916 (2015).

[37] A. C. Felts, M. J. Andrus, E. S. Knowles, P. A. Quintero, A. R. Ahir, O. N. Risset, C. H. Li, I. Maurin, G. J. Halder, K. A. Abboud, M. W. Meisel, and D. R. Talham, J. Phys. Chem. C 120, 5420 (2016).

[38] G. Félix, W. Nicolazzi, L. Salmon, G. Molnar, M. Perrier, G. Maurin, J. Larionova, J. Long, Y. Guari, and A. Bousseksou, Phys. Rev. Lett. 110, 235701 (2013).

[39] G. Félix, W. Nicolazzi, M. Mikolasek, G. Molnar, and A. Bousseksou, Phys. Chem. Chem. Phys. 16, 7358 (2014). 
[40] G. Félix, M. Mikolasek, G. Molnar, W. Nicolazzi, and A. Bousseksou, Chem. Phys. Lett. 607, 10 (2014).

[41] G. Félix, M. Mikolasek, H. Peng, W. Nicolazzi, G. Molnar, A. I. Chumakov, L. Salmon, and A. Bousseksou, Phys. Rev. B 91, 024422 (2015).

[42] M. Paez-Espejo, M. Sy, and K. Boukheddaden, J. Am. Chem. Soc. 138, 3202 (2016).

[43] J. Linares, C. M. Jureschi, A. Boulmaali, and K. Boukheddaden, Physica B: Condensed Matter 486, 164 (2016).

[44] A. Muraoka, K. Boukheddaden, J. Linares, and F. Varret, Phys. Rev. B 84, 054119 (2011).

[45] L. Stoleriu, P. Chakraborty, A. Hauser, A. Stancu, and C. Enachescu, Phys. Rev. B 84, 134102 (2011).

[46] A. Slimani, K. Boukheddaden, and K. Yamashita, Phys. Rev. B 89, 214109 (2014).

[47] K. Boukheddaden, A. Slimani, M. Sy, F. Varret, H. Oubouchou, and R. Traiche, Physical Properties of 2D Spin-Crossover Solids from an Elastonic Description: Effect of Shape, Size and Spin-Distortion Interactions, edited by J.-C. Levy (Pan Stanford, Singapore, 2016), pp. 333-434.

[48] H. Oubouchou, A. Slimani, and K. Boukheddaden, Phys. Rev. B 87, 104104 (2013).

[49] A. Slimani, K. Boukheddaden, F. Varret, H. Oubouchou, M. Nishino, and S. Miyashita, Phys. Rev. B 87, 014111 (2013).
[50] J. Jung, F. Bruchhäuser, R. Feile, H. Spiering, and P. Gütlich, Z Phys. B 100, 517 (1996).

[51] H. Spiering, K. Boukheddaden, J. Linares, and F. Varret, Phys. Rev. B 70, 184106 (2004).

[52] S.-i. Ohkoshi, K. Imoto, Y. Tsunobuchi, and S. T. H. Tokoro, Nat. Chem. 3, 564 (2011).

[53] D. Y. Wu, O. Sato, Y. Einaga, and C. Y. Duan, Angew. Chem. Int. Ed. Engl. 48, 1475 (2009).

[54] T. D. Oke, F. Hontinfinde, and K. Boukheddaden, Eur. Phys. J. B 86, 271 (2013).

[55] K. Boukheddaden, I. Shteto, B. Hoo, and F. Varret, Phys. Rev. B 62, 14796 (2000).

[56] W. C. Yu and P. Gielisse, Mater. Res. Bull. 6, 621 (1971).

[57] A. Bousseksou, J. Nasser, J. Linares, K. Boukheddaden, and F. Varret, J. Phys. I France 2, 1381 (1992).

[58] M. Sorai, Top. Curr. Chem. 235, 153 (2004).

[59] K. Boukheddaden and A. Bailly-Reyre, Eur. Phys. Lett. 103, 26005 (2013).

[60] A. Slimani, K. Boukheddaden, F. Varret, M. Nishino, and S. Miyashita, J. Chem. Phys. 139, 194706 (2013).

[61] A. Slimani, K. Boukheddaden, and K. Yamashita, Phys. Rev. B 92, 014111 (2015).

[62] C. C. Cutler and H. R. Ransom, Another Angle on Acoustic Microscopy (Oblique), edited by L. W. Kessler (Springer, US, 1988), pp. 565-577. 